\title{
End-of-life care in oxygen-dependent COPD and cancer: a national population- based study
}

To the Editor:

Chronic obstructive pulmonary disease (COPD) is the third leading cause of mortality worldwide [1], and is associated with high morbidity and poor symptom control for long periods of time as the disease progresses [2]. At the end of life, COPD patients have a symptom burden comparable to, and often greater than, that associated with cancer [3]. Comparison between COPD and cancer is relevant because cancer patients have well-established palliative care programmes. Despite having extensive and similar end-of-life (EOL) needs to cancer patients, studies report unrelieved symptoms and low referral rates to palliative care in advanced COPD patients [4].

Knowledge about symptoms and symptomatic treatments near death are vital to identify healthcare inequalities, and to identify ways to improve EOL care in advanced COPD. The aim of this study was to estimate the prevalence of symptoms and their management in the last week of life in people with oxygen-dependent COPD or cancer.

This was a nationwide, registry-based cohort study including all patients starting long-term oxygen therapy (LTOT) for physician-diagnosed COPD in the national Swedevox register who died between January 1, 2011 and October 14, 2013. The Swedevox register prospectively includes patients starting LTOT in Sweden with a population-based coverage of $\sim 85 \%$ [5]. Details of the register are described elsewhere [6].

Data on people with oxygen-dependent COPD were cross-linked using each patient's unique Swedish identification number with data in the Swedish Register of Palliative Care (SRPC). Patients in SRPC who died from cancer during the same time period were included as the comparator group. SRPC is a national quality register of the care of patients during their last week of life regardless of place of care or diagnosis, with a coverage of $87.4 \%$ of all cancer deaths nationwide in 2013 [7].

The SRPC collects data through an end-of-life questionnaire (ELQ) completed retrospectively by the responsible nurse and/or physician within a week of the patient's death, based on the patient record and experience of the care that may not have been documented, preferably after a team discussion, therefore including the experience of all team members. The ELQ includes data on the presence of breathlessness, pain, death rattle, nausea, anxiety and confusion, and prevalence of prescribing "as-needed" medications for pain, nausea, anxiety and death rattle during the last 7 days of life. A previous study supported the validity of the ELQ [8].

Prevalence was considered for each symptom in the questions of the ELQ: "Were any of the following symptoms prevalent at some time during the last week of life?" (yes or no). For any reported symptom, the level of symptom relief was graded as relieved, partially relieved or unrelieved. Prevalence of as-needed medication prescriptions was analysed among symptomatic patients for each identified symptom according to the question "Was medication prescribed for use 'as needed' in the form of injections before death for pain, death rattle, nausea and anxiety?" (yes or no).

The study was approved by local ethics committee (LundDNr 2013/379; University of Lund, Lund, Sweden). Statistical methods included standard descriptive statistics, t-tests and chi-square tests as appropriate, using Stata version 12 (StataCorp LP, College Station, TX, USA).

A total of 1128 COPD patients (mean \pm SD age $78 \pm 8$ years at death; $60 \%$ women) and 56843 cancer patients (age $75 \pm 12$ years at death; $49 \%$ women) were included. Of LTOT patients from Swedevox, 59\% were registered in SPRC and included in the study. Characteristics were similar between LTOT patients who were and were not included in terms of age, sex, lung function, body mass index and arterial blood gas levels, supporting the external validity of the study.

Compared with patients with cancer, COPD patients suffered from more breathlessness (73\% versus 22\%) and anxiety (63\% versus $54 \%$ ) but less pain ( $52 \%$ versus $81 \%)$ and nausea ( $11 \%$ versus $23 \%)$ during the last 
week of life (figure 1a). COPD patients had lower rates of complete relief from breathlessness (22\% versus $37 \%)$, anxiety (52\% versus $61 \%)$ and death rattle (33\% versus $44 \%)$ than cancer patients (figure $1 \mathrm{~b}$ ) $(\mathrm{p}<0.001$ for all comparisons).

Prescription of as-needed medications during the last week of life was significantly lower in COPD than cancer for all symptoms: anxiety (76\% versus $89 \%)$, death rattle (75\% versus $88 \%)$, pain ( $80 \%$ versus $96 \%)$ and nausea ( $46 \%$ versus $77 \%)$ ( $\mathrm{p}<0.001$ for all comparisons). Furthermore, COPD patients had fewer as-needed medications prescribed to address specific symptoms when these were recorded: for anxiety ( $82 \%$ versus $95 \%)$, death rattle ( $89 \%$ versus $95 \%)$, pain (93\% versus $97 \%$ ) and nausea (64\% versus $89 \%)$ $(\mathrm{p}<0.001$ for all comparisons).

To evaluate the robustness of the findings, we compared symptom prevalence among patients for whom the symptom severity was rated according to the ELQ using a validated instrument. Results were similar for all symptoms, except that the relief of pain was higher in both groups, which supports the robustness of the findings.

Death was expected among $80 \%$ of COPD patients and 95\% of cancer patients. However, COPD patients more often died in hospital (49\% versus $28 \%$ ) and received less specialised palliative home care (4\% versus $14 \%)$. Fewer COPD patients had an EOL discussion (33\% versus $61 \%)(\mathrm{p}<0.001$ for all comparisons).

Our study shows that patients with oxygen-dependent COPD suffered from a high symptom load with poor symptom control in the last week of life. Breathlessness was three times more common in oxygen-dependent COPD than cancer patients. Breathlessness was fully relieved for only $22 \%$ of patients. Prescription of as-needed medications for all symptoms was significantly lower among COPD patients than in patients with cancer.

These findings are in line with previous reports that COPD patients suffer from a high burden of symptoms, especially breathlessness, at the end of life [9]. We have expanded previous findings with data on prescription of palliative medications during the last week in advanced COPD specifically and comparing it with a cancer cohort that usually receives higher-quality EOL care.

Another finding is that COPD patients still have limited access to specialised care services and receive less palliative care than patients with cancer, consistent with previous reports $[10,11]$. The limited specialised health and social care received by most COPD patients highlights the unequal distribution of specialised resources at the end of life depending on the underlying disease.

Strengths of the present study include its national population-based design, and inclusion of the largest cohort to date of patients with oxygen-dependent COPD and cancer. Few previous studies included the very last days of life and most studies were old or involved small cohorts [10, 12]. We report population-based, real-world data, and the findings probably have high external validity due to the national setup.

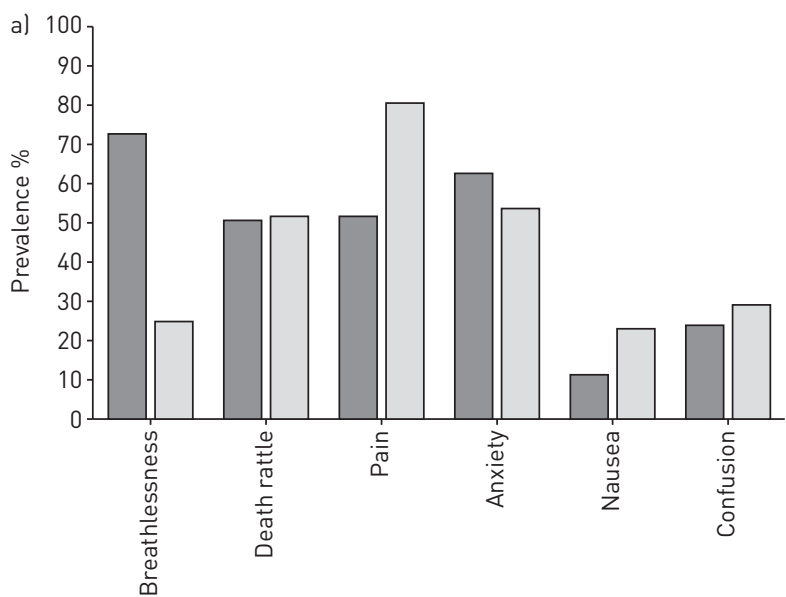

COPD

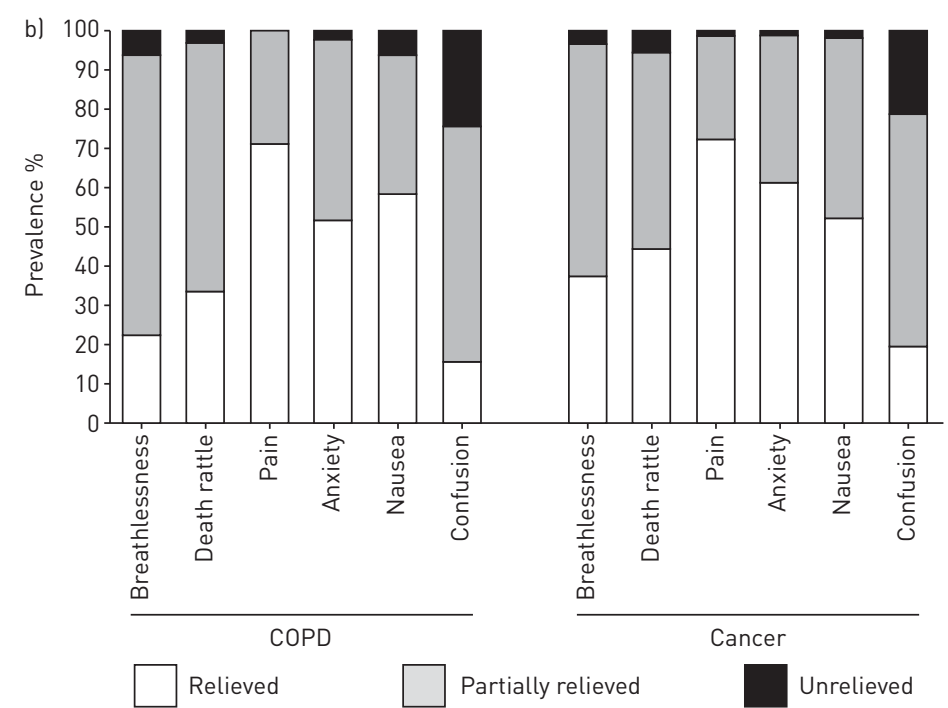

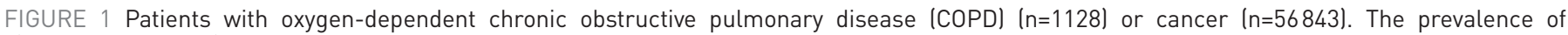
a) symptoms, and b) relieved, partially relieved and unrelieved symptoms in symptomatic patients during the last week of life in Sweden. 
Limitations of our study include that data on palliative care in SRPC were collected retrospectively. The difficulties of predicting the prognosis in advanced COPD would make prospective data collection near death problematic among severely ill patients. The ELQ was answered by staff, which could be affected by recall or reporting bias. However, staff proxy assessments of symptoms in patients with advanced disease might be a valid alternative, especially for the detection of breathlessness [13].

For clinicians, this study highlights the need for adequate EOL care among patients with advanced COPD. This includes symptom management, the need for integrated respiratory and palliative care, and increased access to specialised palliative care services [14]. Breathlessness is very common in advanced COPD and poor symptom control remains a significant problem before death. At present, there is substantial evidence in favour of treatment with opioids, as randomised trials have shown that oral sustained-release morphine can relieve chronic refractory breathlessness [15]. A recent study by our group supports the safety of low-dose opioids in severe COPD [16].

In conclusion, this study identifies areas for improvement of the quality of EOL care received by COPD patients. Further engagement of health service providers and policy makers is necessary in order to provide these patients with equal and decent EOL care.

@ERSpublications

COPD patients have a high symptom burden but receive less palliative care than cancer patients at the end of life http://ow.ly/NchLv Zainab Ahmadi ${ }^{1}$, Staffan Lundström ${ }^{2,3}$, Christer Janson ${ }^{4}$, Peter Strang ${ }^{2,3}$, Margareta Emtner ${ }^{4}$, David C. Currow
and Magnus Ekström
${ }^{1,5}$ Department of Clinical Sciences, Division of Respiratory Medicine and Allergology, Lund University Hospital, Lund,
Sweden. ${ }^{2}$ Palliative Care Services, Stockholms Sjukhem Foundation, Stockholm, Sweden. ${ }^{3}$ Department of Oncology-
Pathology, Karolinska Institute, Stockholm, Sweden. ${ }^{4}$ Department of Medical Sciences, Respiratory Medicine and
Allergology, Uppsala University, Uppsala, Sweden. ${ }^{5}$ Discipline, Palliative and Supportive Services, Flinders University, Adelaide, Australia.

Correspondence: Zainab Ahmadi, Department of Clinical Sciences, Division of Respiratory Medicine and Allergology, Lund University Hospital, SE- 22100 Lund, Sweden. E-mail: zai.ahmd@gmail.com

Received: Jan 192015 | Accepted after revision: May 162015 | First published online: August 62015

Conflict of interest: None declared.

Support statement: This study was funded by the Swedish Society of Medicine, the Swedish Respiratory Society, the Scientific Committee of Blekinge County Council, the Wera and Emil Cornell Foundation, and the Swedish Heart-Lung Foundation.

Acknowledgements: The work was carried out at the Department of Respiratory Medicine, Blekinge Hospital, Karlskrona, and Lund University, Lund, Sweden. We thank Bengt Midgren (Department of Clinical Sciences, Division of Respiratory Medicine and Allergology, Lund University Hospital, Lund, Sweden) for his valuable insights, Greger Fransson (Palliative Care Services, Stockholms Sjukhem Foundation, Stockholm, Sweden) for database management, and all the physicians and nurses who collected the data and cared for the patients.

\section{References}

1 Lozano R, Naghavi M, Foreman K, et al. Global and regional mortality from 235 causes of death for 20 age groups in 1990 and 2010: a systematic analysis for the Global Burden of Disease Study 2010. Lancet 2012; 380: 2095-2128.

2 Currow DC, Smith J, Davidson PM, et al. Do the trajectories of dyspnea differ in prevalence and intensity by diagnosis at the end of life? A consecutive cohort study. J Pain Symptom Manage 2010; 39: 680-690.

3 Bausewein C, Booth S, Gysels M, et al. Understanding breathlessness: cross-sectional comparison of symptom burden and palliative care needs in chronic obstructive pulmonary disease and cancer. J Palliat Med 2010; 13: $1109-1118$.

4 Luddington L, Cox S, Higginson I, et al. The need for palliative care for patients with non-cancer diseases: a review of the evidence. Int J Palliat Nurs 2001; 7: 221-226.

5 Strom K, Boe J. A national register for long-term oxygen therapy in chronic hypoxia: preliminary results. Eur Respir J 1988; 1: 952-958.

6 Ekstrom MP, Hermansson AB, Strom KE. Effects of cardiovascular drugs on mortality in severe chronic obstructive pulmonary disease. Am J Respir Crit Care Med 2013; 187: 715-720.

7 The Swedish Register of Palliative Care. Årsrapport för Svenska Palliativregistret verksamhetsåret 2013 [Annual report of the Swedish Register of Palliative Care for the fiscal year 2013]. http://palliativ.se/wp-content/uploads/ 2014/03/SvenskaPalliatvregistret2014.pdf Date last accessed: September 1, 2014. Date last updated: 2014.

8 Martinsson L, Heedman PA, Lundstrom S, et al. Validation study of an end-of-life questionnaire from the Swedish Register of Palliative Care. Acta Oncol 2011; 50: 642-647.

9 Moens K, Higginson IJ, Harding R. Are there differences in the prevalence of palliative care-related problems in people living with advanced cancer and eight non-cancer conditions? A systematic review. J Pain Symptom Manage 2014; 48: 660-77. 
10 Edmonds P, Karlsen S, Khan S, et al. A comparison of the palliative care needs of patients dying from chronic respiratory diseases and lung cancer. Palliat Med 2001; 15: 287-295.

11 Elkington $\mathrm{H}$, White $\mathrm{P}$, Addington-Hall J, et al. The healthcare needs of chronic obstructive pulmonary disease patients in the last year of life. Palliat Med 2005; 19: 485-491.

12 Claessens MT, Lynn J, Zhong Z, et al. Dying with lung cancer or chronic obstructive pulmonary disease: insights from SUPPORT. Study to Understand Prognoses and Preferences for Outcomes and Risks of Treatments. J Am Geriatr Soc 2000; 48: Suppl., S146-S153.

13 Simon ST, Altfelder N, Alt-Epping B, et al. Is breathlessness what the professional says it is? Analysis of patient and professionals' assessments from a German nationwide register. Support Care Cancer 2014; 22: 1825-1832.

14 Higginson IJ, Bausewein C, Reilly CC, et al. An integrated palliative and respiratory care service for patients with advanced disease and refractory breathlessness: a randomised controlled trial. Lancet Respir Med 2014; 2: 979-987.

15 Abernethy AP, Currow DC, Frith P, et al. Randomised, double blind, placebo controlled crossover trial of sustained release morphine for the management of refractory dyspnoea. BMJ 2003; 327: 523-528.

16 Ekstrom MP, Bornefalk-Hermansson A, Abernethy AP, et al. Safety of benzodiazepines and opioids in very severe respiratory disease: national prospective study. BMJ 2014; 348: g445.

\section{Effects of ageing and smoking on pulmonary computed tomography scans using parametric response mapping}

To the Editor:

Chronic obstructive pulmonary disease (COPD) is an obstructive lung disease often caused by cigarette smoke, and characterised by inflammation and abnormalities of the large and small airways (i.e. those with an internal diameter $<2 \mathrm{~mm}$ ), as well as by alveolar destruction (emphysema). Recent evidence suggests that small airway disease precedes emphysema [1] and, therefore, it may be useful to identify the presence and extent of small airway disease and emphysema in early COPD, or preferably, even before the onset of disease.

Parametric response mapping (PRM) is a novel technique to analyse pulmonary computed tomography (CT) scans in order to quantify the extent of small airway disease $\left(\mathrm{PRM}^{\mathrm{fSAD}}\right)$, emphysema $\left(\mathrm{PRM}^{\mathrm{Emph}}\right.$ ) and parenchymal disease $\left(\mathrm{PRM}^{\mathrm{PD}}\right)$, the latter reflecting increased attenuation of normal lung parenchyma $[2,3]$. We aimed to evaluate the PRM technique in a cohort of well-characterised, respiratory-healthy subjects with a wide age range. As smoking and ageing are both risk factors in the development of COPD [4], we hypothesised that 1) an older age is associated with more $\mathrm{PRM}^{\mathrm{fSAD}}, \mathrm{PRM}^{\mathrm{Emph}}$ and $\mathrm{PRM}^{\mathrm{PD}}$, and 2) current smoking is associated with more $\mathrm{PRM}^{\mathrm{fSAD}}, \mathrm{PRM}^{\mathrm{Emph}}$ and $\mathrm{PRM}^{\mathrm{PD}}$. Finally, we investigated the association between PRM measurements and pulmonary function measurements.

We selected current smokers and never-smokers older than 18 years, without respiratory symptoms and with no history of respiratory diseases. In addition, they had normal pulmonary function, defined as a post-bronchodilator forced expiratory volume in $1 \mathrm{~s}(\mathrm{FEV} 1) /$ forced vital capacity (FVC) ratio above the lower limit of normal, no bronchial hyperresponsiveness and reversibility of FEV 1 to salbutamol $<10 \%$ of the predicted value.

Spirometry (FEV1, FVC, FEV1/FVC and forced expiratory flow at 25-75\% of FVC (FEF25-75\%)), body plethysmography (residual volume (RV), total lung capacity (TLC) and RV/TLC) and methacholine provocation tests were performed according to international guidelines $[5,6]$. Transfer factor of the lung for carbon monoxide corrected from haemoglobin (TLCOc) adjusted for alveolar volume (VA) was measured using the single breath-holding technique, and small airway resistance (resistance at $5 \mathrm{~Hz}(R 5)$ minus resistance at $\left.20 \mathrm{~Hz}\left(R_{20}\right)\right)$ and reactance at $5 \mathrm{~Hz}\left(X_{5}\right)$ were measured by impulse oscillometry. We considered $\mathrm{FEF}_{25}-75 \%, \mathrm{FEF} 25-75 \% / \mathrm{FVC}, \mathrm{RV} / \mathrm{TLC}, R 5-R 20$ and $X_{5}$ as small airway measurements.

Thin-slice (i.e. 75-mm) pulmonary CT scans were made at full in- and expiration (RV). PRM was performed to quantify $\mathrm{PRM}^{\mathrm{fSAD}}, \mathrm{PRM}^{\mathrm{Emph}}$ and $\mathrm{PRM}^{\mathrm{PD}}$ as percentage of total lung volume, as described previously [2,3]. We applied linear regression analyses to assess associations between both age and smoking, and $\mathrm{PRM}^{\mathrm{fSAD}}, \mathrm{PRM}^{\mathrm{Emph}}$ and $\mathrm{PRM}^{\mathrm{PD}}$, adjusted for sex. Next, we performed linear regression 